\title{
La historia se repite: grandes retos para gobiernos débiles
}

\author{
History repeats itself: great challenges for weak governments
}

Luis Hernando Portillo-Riascos ${ }^{1 *}$ orcid.org/0000-0003-3362-6934

1. Departamento de Economía de la Universidad de Nariño. Pasto, Colombia

Fecha de recepción: Junio 18 - 2020

Fecha de revisión: Septiembre 15 - 2020

Fecha de aceptación: Diciembre 04 - 2020

Portillo-Riascos LH. La historia se repite: grandes retos para gobiernos débiles. Univ. Salud. Suplemento1: Especial Covid-19. 2020 22(3):315326. DOI: https://doi.org/10.22267/rus.202203.204

\section{Resumen}

Introducción: COVID-19 representa un fenómeno que no tiene precedentes en la historia mundial reciente. Ante esta situación, se requiere de una fuerte intervención estatal que permita mitigar los efectos socioeconómicos que hasta el momento se han causado. Objetivo: Evaluar las medidas adoptadas por el gobierno nacional colombiano y las entidades territoriales del departamento de Nariño, ante los desafíos sociales y económicos asociados a COVID-19. Materiales y métodos: Revisión documental, a partir de informes gubernamentales, reportes divulgados por institutos de investigación nacional e internacional, publicaciones de prensa, y las bases de datos Web of Science y Science Direct. Resultados: Se estructuró la información de las disposiciones fiscales que tomaron los distintos niveles de gobierno para atender las obligaciones derivadas de COVID-19 durante el periodo marzo-mayo de 2020. Conclusiones: Tanto el Gobierno Nacional como las autoridades territoriales del departamento de Nariño han tenido que asumir inversiones extraordinarias para atender los gastos demandados para la atención de la pandemia por COVID-19. Sin embargo, dichas medidas se muestran insuficientes y tardías para el tamaño de los efectos que se han generado en el marco de la emergencia sanitaria.

Palabras clave: COVID-19; política pública; economía; salud pública; Colombia. (Fuente: DeCS, Bireme).

\begin{abstract}
Introduction: COVID-19 represents an unprecedented phenomenon in recent human history. This new situation requires a strong official intervention to mitigate the socioeconomic impact caused to date. Objective: To assess the measures adopted by the Colombian government and official institutions of the Nariño Department (State) facing the social and economic challenges associated with COVID-19. Materials and methods: A documentary review based on government data, reports released by national and international research institutes, press communications and the Web of Science and Science Direct databases. Results: Information from fiscal administrative acts promulgated by different government entities to meet the obligations derived from COVID-19 during the March-May 2020 period was structured. Conclusions: Both the Colombian national government and local authorities from the Nariño Department have had to assume extraordinary expenditures to confront the costs associated with the COVID-19 pandemic. However, these measures seem insufficient and belated in the face of the magnitude of the effects caused by the pandemic.
\end{abstract}

Key words: COVID-19; public policy; economics; public health; Colombia. (Source: DeCS, Bireme).

\footnotetext{
*Autor de correspondencia

Luis Hernando Portillo Riascos

e-mail: luishernando@udenar.edu.co
} 


\section{Introducción}

Los efectos de la pandemia por COVID-19 representan un fenómeno que no tiene precedentes en la historia mundial reciente, cuyos efectos en la salud pública y en las dimensiones social y económica se empezaron a visualizar desde finales de 2019. Efectivamente, las pandemias han tenido lugar en muchos momentos ${ }^{(1)}$, no obstante, hay acuerdo en que la situación derivada por COVID-19 tiene una serie de particularidades que hacen que no se pueda establecer un parangón(2,3).

En primer lugar, en la dimensión social y aunque en distintos niveles y con ciertas diferencias temporales, todos los países se han visto afectados por COVID-19, casi de manera simultánea(4); situación que no se había presentado en condiciones de guerra, que perjudicaban especialmente a aquellos países o regiones que entraban en conflicto, a pesar de los efectos indirectos que ocasionaban(3).

En lo económico sucede algo similar, por ejemplo, en los episodios de crisis financiera internacional, como el ocurrido en 2008-2009, el mayor impacto se dio en determinados países, sin que se haya dado un "efecto de contagio" tan amplio y prácticamente sincrónico(4,5).

En segundo lugar, esta pandemia ha desatado sus efectos en un momento en que la economía mundial muestra un alto nivel de integración en diferentes ámbitos (comercial, financiero, etc.). Dicho fenómeno, que inicialmente se ha considerado como algo positivo, en la actualidad está actuando en contra del objetivo de contener el contagio (1).

Un tercer elemento es que la crisis no se origina propiamente en la economía, sino que se trata de un problema de salud pública, cuyos efectos se trasladan inmediatamente a la economía es decir, se encuentra en un estado de coma inducido, como lo refiere Paul Krugman(6). En este contexto, lo importante es saber que existe una crisis social y económica de gran magnitud que requiere enfrentarla de la mejor manera posible.

La capacidad de intervención que tienen los gobiernos depende de muchos factores, entre estos, del espacio fiscal, es decir, la disponibilidad de recursos con que cuentan los estados para apoyar tanto a las familias como a las empresas. Asimismo, la posibilidad de intervención del Estado no solamente es diferente entre países, sino también entre departamentos y municipios, incluso entre niveles de gobierno(7).

El cuarto elemento está relacionado con que al desatarse el problema económico, se presenta una crisis simultánea tanto en la oferta como en la demanda agregada(8), situación bastante singular. En el caso de las empresas, las dificultades se asocian a la incapacidad de producir y comercializar los bienes y servicios, debido a las restricciones del confinamiento en la mayoría de países. En el caso de la demanda, las familias se han visto en la necesidad de modificar radicalmente sus decisiones de consumo en un periodo de tiempo muy corto, dado que muchas de ellas han perdido un volumen importante de sus ingresos. En las dos situaciones, la incertidumbre genera un efecto negativo, no solamente desde el punto de vista social, sino también en la dinámica económica(4).

En este punto, se argumenta que la debilidad institucional o vulnerabilidad de los países en desarrollo ante los choques externos no es el algo nuevo ${ }^{(9)}$ y, por el contrario, ha sido un suceso recurrente(10). En este sentido, se sostiene que si bien es cierto que la pandemia de COVID-19 es reciente, lo llamativo es la debilidad del Estado para responder ante este tipo de desafíos(10). Dadas estas circunstancias, lo que se aduce es que la crisis social y económica derivada de esta pandemia va a ocasionar una serie de secuelas bastante profundas que van a tardar mucho tiempo en atenuarse.

Bajo el marco expuesto, el objetivo de este estudio es evaluar las medidas adoptadas tanto por el gobierno nacional colombiano como por las entidades territoriales del departamento de Nariño, ante los desafíos sociales y económicos asociados a la pandemia por COVID-19.

\section{Materiales y métodos}

Se examinó la dimensión que tiene el fenómeno de la pandemia por COVID-19 y la capacidad de respuesta de los gobiernos, en el ámbito nacional y local, para enfrentar las consecuencias derivadas de este hecho.

Es importante tener en cuenta que la información utilizada para el desarrollo del presente estudio, es secundaria y corresponde a un evento en curso. Por 
tanto, no se dispone de un acervo de estudios previos, así como sucede en el aspecto económico, los balances realizados se asumen con un carácter provisional, por cuanto los datos definitivos sobre el comportamiento de muchos de los agregados macroeconómicos presentan rezagos temporales.

Se realizó una revisión documental de informes gubernamentales, reportes divulgados por institutos de investigación nacionales e internacionales, publicaciones de prensa, y las bases de datos Scopus, Web of Science y Science Direct. Dichos informes evalúan las medidas adoptadas tanto por el Gobierno Nacional como por las entidades territoriales (gobernaciones y alcaldías) ante la emergencia desatada por la pandemia de COVID-19 durante el periodo marzo-mayo de 2020. Para el caso del departamento de Nariño, se tomó la información de contratación publicada por entidades estatales entre marzo y abril de 2020, relacionada con la destinación de recursos destinados para atender gastos derivados de la crisis desencadenada por la COVID-19. Dicha información está disponible en el sistema Electrónico para la Contratación Pública (SECOP)(11).

\section{Resultados}

Después de haber realizado algunas reflexiones iniciales, se describen las medidas económicas más importantes adoptadas tanto por el Gobierno Nacional como por las entidades territoriales en el departamento de Nariño para enfrentar la pandemia de COVID-19. Es probable que una parte de la información no se relacione dentro de esta investigación, por dos razones: a) porque no se publican todas las cifras, y b), porque los datos son preliminares, entendiendo que día a día se toman decisiones en las distintas dimensiones. Asimismo, existen divergencias importantes entre las cifras publicadas por las diferentes instituciones, lo cual se explica por los contrastes que existen a la hora de hacer las proyecciones, y los supuestos incorporados en cada caso. Además, hay algunos conceptos que no son efectivos, como las garantías crediticias o avales que entregan los gobiernos para soportar las operaciones de financiamiento, fundamentalmente dirigidas a las empresas.

En la primera sección se realiza un comparativo entre el monto económico invertido por Colombia para la atención de la pandemia de COVID-19, con relación a la inversión realizada por otros países sudamericanos. En la segunda parte se exponen las principales medidas fiscales que ha tomado el Gobierno Nacional colombiano. En la tercera sección se hace un breve análisis de las disposiciones adoptadas por los gobiernos territoriales en el departamento de Nariño.

\section{A. ¿Cuál es el costo económico de la crisis causada por la COVID-19 para los países sudamericanos?}

Evidentemente, lo más importante de la crisis derivada de COVID-19 son los efectos sociales. No obstante, es interesante examinar la capacidad de respuesta de los gobiernos de los diferentes países para atender la problemática asociada a dicho fenómeno, y el costo que esto representa para el erario.

Con relación al comportamiento del PIB (Producto Interno Bruto), la CEPAL(Comisión Económica para América Latina y el Caribe )(12),-estima una caída del 9,1\% para América Latina y el Caribe para el año 2020. En el caso de América del Sur, se proyecta una reducción del PIB $(-9,4 \%)$, porcentaje que está sobre la media latinoamericana. Sin embargo, hay diferencias importantes entre países, así, mientras Venezuela, Perú, Argentina y Brasil muestran un impacto mayor de la crisis, con unas tasas estimadas del $-26 \%,-6,5 \%,-10,5 \%$ y $-9.2 \%$, respectivamente, los países que salen "mejor librados" de esta situación son Paraguay, Uruguay, Bolivia y Colombia, con unos porcentajes del $-2,3 \%, \quad-5 \%, \quad-5,2 \%$ y $\quad-5,6 \%$, respectivamente.

Las estimaciones que hace el Banco Mundial en el informe de Perspectivas Económicas globales, publicado en junio de 2020, también son pesimistas, se proyecta una caída del PIB mundial del 5,2\%, que para las economías desarrolladas alcanzaría el 7\%, y para los países emergentes sería del 2,5\%. En el caso de las economías latinoamericanas, la contracción del PIB se estima en un $7,2 \%{ }^{(13)}$.

La situación económica actual supone la caída más significativa del PIB en las últimas décadas en el contexto mundial, comparable incluso con la Gran Depresión de 1929(14). Para el caso de América Latina y el Caribe, las proyecciones del comportamiento del PIB apuntan a un escenario aún peor que el de la década de los ochenta, considerado como uno de los periodos más adversos en la historia reciente de la

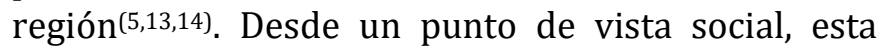


emergencia agudiza los problemas estructurales de este tipo de países, especialmente por los altos niveles de pobreza, desigualdad, informalidad y las debilidades en los sistemas de asistencia social, incluido el sanitario(4,15). Esta situación se hace más compleja si se tiene en cuenta las limitaciones fiscales de los gobiernos en el propósito de mitigar las consecuencias derivadas de la crisis social.

Una vez se resumieron los pronósticos del comportamiento del PIB, en adelante, se hace una comparación de los estimativos del costo fiscal en el cual habrían incurrido los países latinoamericanos durante la fase inicial de la pandemia. De acuerdo con la CEPAL(5), a principios del mes de abril de 2020, la intervención fiscal de estos países se habría ubicado en un rango que está entre el 0,6\% y el 12\% del PIB. Naturalmente, hay que tener en cuenta que durante los meses siguientes se ampliaron las medidas, aumentando con ello el costo fiscal. De todas maneras, con estas cifras, se pueden hacer comparaciones.

Para el caso de Colombia, la CEPAL(5) estimó que las medidas gubernamentales adoptadas para atender la emergencia sanitaria habrían generado un costo fiscal cercano al 1,4\% del PIB (14 billones de pesos colombianos aproximadamente). Dicho monto se encuentra por debajo de las proyecciones realizadas para países como Brasil (5,8\% del PIB), Perú (7\% del PIB) y Chile $(5,5 \%)$. Ávila llega a una conclusión similar, advirtiendo que los paquetes de ayudas entregados por el gobierno en Colombia solamente superan a México, Barbados, Venezuela y Ecuador(16).

\section{B. La intervención del gobierno colombiano frente a la pandemia de COVID-19}

El propósito de esta sección es describir, de manera general, las medidas tomadas por el Gobierno colombiano dirigidas a atender la emergencia sanitaria durante el periodo marzo-mayo de 2020. Es importante mencionar que en Colombia existen dos niveles de gobierno: el Gobierno Nacional Central (GNC) y los gobiernos territoriales, constituidos por las gobernaciones y los municipios, como se presenta a continuación.

\section{Medidas adoptadas por el GNC}

Son varias las decisiones tomadas por el GNC en el marco del estado de emergencia sanitaria, económica y social, decretada por el presidente de la República a partir del 17 de marzo de 2020. Se describe por separado aquellas disposiciones dirigidas a la atención de familias y a las empresas. Además, se diferencia las medidas existentes previas a la pandemia, de las que surgieron en razón a la misma.

Con relación a las ayudas entregadas a las familias, estas se pueden clasificar en dos grupos. En primer lugar, los programas sociales que ya vienen funcionando en Colombia desde hace varios años, tales como Familias en Acción, Jóvenes en Acción y Colombia Mayor. Y en segundo lugar, los programas nuevos, entre los que se incluye la compensación de Impuesto sobre el Valor Agregado (IVA) y el Ingreso Solidario. El costo de dichas ayudas hasta el mes de abril habría ascendido a un monto aproximado de 4 billones pesos $(0,4 \% \text { del PIB })^{(17)}$. No obstante, en el mes de mayo e inicios de junio de 2020, el Gobierno Nacional tomó la decisión de ampliar el monto pagado y la duración de las transferencias monetarias(18).

Dentro de las medidas adoptadas por el GNC para atender a las familias, también se contemplan los aportes en especie, las transferencias a los cesantes y a las personas a quienes se les suspendió o terminó el contrato de trabajo, y las medidas que se pueden considerar como no monetarias, que están relacionadas con las disposiciones en materia de arriendos, facilidades crediticias, pago de servicios públicos, etc.(17).

En el caso de las empresas, algunas de las ayudas gubernamentales que se han entregado hasta el momento son: garantías crediticias para facilitar el financiamiento por parte del sistema bancario, reducción temporal de la carga de seguridad social (aportes a pensiones y parafiscales), exención del IVA e impuesto al consumo para algunos sectores, la eliminación temporal de la obligación de pagar algunos impuestos por anticipado, la devolución automática de saldos a favor en renta e IVA, subsidio del $50 \%$ de la prima de servicios y el subsidio transitorio a la nómina(17).

A continuación, se resumen las medidas más importantes que el Gobierno Nacional ha adoptado hasta el momento para financiar los compromisos que está adquiriendo para afrontar la emergencia sanitaria. Es importante advertir que los montos son preliminares, tanto por el recaudo efectivo que se alcance en algunos de los conceptos, como por la vigencia de las medidas. 
Los recursos necesarios para financiar los gastos derivados de la pandemia provienen de las siguientes fuentes(16,17):

- $\$ 12,1$ billones de los ahorros generados en el Fondo de Ahorro y Estabilización (FAE), creado mediante la Ley 1530 de 2012.

- $\quad \$ 3,8$ billones de los aportes al Fondo Nacional de Pensiones de las Entidades Territoriales (FONPET).

- Contribución especial de los funcionarios públicos, en aquellos casos en los que los ingresos mensuales superen los diez millones de pesos. Por este mecanismo, se espera recaudar una suma de $\$ 395$ mil millones. Sin embargo, en el mes de agosto de 2020, la Corte Constitucional declaró inconstitucional el cobro de este tributo(19).

- $\$ 9,8$ billones correspondientes a inversiones forzosas en títulos de solidaridad por parte del sector financiero.

- $\$ 48$ billones provenientes de los recursos de capital de las entidades del Estado (Holding financiero) destinados a reforzar las garantías otorgadas en productos de créditos a las empresas.

- Traslado de pensionados de los fondos privados de pensiones a la Administradora Colombiana de Pensiones (COLPENSIONES). Esta decisión le permitiría ahorrar el GNC un monto aproximado de 5 billones de pesos. No obstante, en el mes de julio de 2020, la Corte Constitucional descartó esta disposición (20).

La necesidad de recurrir a este tipo de fondos se explica, en primer lugar, por la contingencia de la emergencia sanitaria, que implica que estos gastos no se encuentran apropiados en el presupuesto nacional ni en el de los gobiernos territoriales. Por tal motivo, se tiene que recurrir a fuentes adicionales.

En segundo lugar, esta situación también se relaciona con el desajuste en las finanzas públicas a partir del crecimiento del gasto(18). De hecho, al final de 2020, se proyecta un nivel del déficit fiscal del 8,2\% del PIB(18). A esto se suman las dificultades fiscales ya existentes, explicadas fundamentalmente por el impacto fiscal generado por la reducción de los precios del petróleo durante el mismo año; particularmente durante los meses de marzo y abril.

\section{La gestión fiscal de la pandemia por parte de las entidades territoriales en el departamento de Nariño}

Los gobiernos territoriales colombianos, a diferencia de lo que sucede en otros países, no tienen las herramientas fiscales necesarias para actuar de una manera ágil y oportuna frente a una problemática de la magnitud de COVID-19(9). En este sentido, una de las principales dificultades es la escasa capacidad para incrementar el recaudo de rentas propias(7). Esta situación también se refleja en el hecho de que la mayoría de los municipios exhibe una dependencia muy significativa con relación a los recursos girados a través del Sistema General de Participaciones (SGP), creado mediante la Ley 715 de 2001 y modificado por la Ley 1176 de 2007. Asimismo, es importante tener en cuenta que la mayor parte de los recursos provenientes del SGP tienen destinación sectorial específica, lo que implica que dichas asignaciones, en su mayoría, no pueden emplearse para atender este tipo de emergencias(7).

Por lo anterior, solamente las grandes ciudades tienen la posibilidad de implementar medidas expansivas de gasto público. Por ejemplo, en el caso de Bogotá, la Alcaldía decidió subsidiar a 350 mil familias con un pago de 423 mil pesos, cuyo propósito fue cubrir los 23 días correspondientes al plazo establecido en la primera cuarentena, que iba desde del 15 de marzo al 12 de abril de 2020. Si se compara la situación de esta ciudad con relación a los municipios de sexta categoría, que son la gran mayoría, la diferencia es abismal.

En este contexto, la capacidad de intervención de los gobiernos territoriales en el marco de la pandemia de COVID-19 es mínima. Solamente podrían utilizar, en el caso extremo, las asignaciones que hacen parte del Propósito General. Sin embargo, en la realidad, esto es imposible, en primer lugar, porque hay otros sectores que se deben atender. Y, en segundo lugar, porque el flujo de recursos se presenta de manera periódica.

\section{¿Cuánto ha invertido Nariño para enfrentar la pandemia por COVID-19?}

Más allá de los recursos invertidos por el Gobierno Nacional, en este estudio se hace un estimativo de la inversión realizada tanto por la Gobernación de Nariño como por los municipios para atender las obligaciones derivadas de la emergencia sanitaria. El monto real, como se entenderá, es muy complejo de 
conocerlo. No obstante, se trata de hacer una aproximación a este tema. Los datos se obtuvieron del Sistema Electrónico de Contratación Pública (SECOP)(11), a partir de la información contractual reportada por las entidades territoriales del departamento de Nariño entre el 2 de febrero y el 23 de abril de 2020 .

Para el caso de los municipios, se seleccionaron 116 contratos, que se encuentran clasificados en el SECOP en el grupo E (alimentos, bebidas y tabaco). Como resultado de este ejercicio, se encontró que las entidades territoriales del departamento de Nariño invirtieron un total de \$ 6308 millones; lo que representa una asignación per cápita de \$7052. Además, se resalta que de los 64 municipios de Nariño, solamente 47 realizaron algún tipo de inversión de esta naturaleza.

También se analizaron los contratos cuyo propósito fue adquirir insumos hospitalarios y dispositivos médicos. En este caso, se identificaron 54 contratos suscritos tanto por parte de los municipios como por la Gobernación de Nariño. El monto total de dichos contratos ascendió a \$ 8316 millones. Sobre esta suma, es importante advertir que el $90 \%$ de estos recursos fueron aportados por la Gobernación de Nariño. No obstante, si se incluye el monto invertido por las Instituciones Prestadoras de Salud (IPS) públicas, la cuantía asciende a un aproximado de 19000 millones de pesos.

En la Tabla 1 se discriminan los contratos firmados por la Gobernación de Nariño para atender la contingencia de COVID-19. En total, se analizaron 21 contratos. Como se puede observar, el porcentaje más alto de las inversiones se destinó a la adquisición de dispositivos médicos (50\%) y a gastos clasificados como ayuda humanitaria (15\%).

Los gobiernos territoriales, incluyendo el Departamento y los municipios, cuentan con los recursos de regalías correspondientes al bienio 20192020. Al respecto, el Gobierno Nacional, en el marco del estado de emergencia sanitaria, expidió el Decreto 513 del 2 de abril de 2020(21), que permite que estos recursos se utilicen para atender las obligaciones asociadas a la pandemia.

En el caso del departamento de Nariño, el uso de los recursos de regalías ha sido prácticamente nulo. Así, a 12 de junio de 2020, entre los 64 municipios y la
Gobernación de Nariño tenían pendiente por comprometer 386000 millones de pesos, de los cuales, la Gobernación registraba un saldo de 315000 millones de $\operatorname{pesos}^{(22)}$.

Tabla 1. Inversión de la Gobernación de Nariño en la atención del COVID-19

\begin{tabular}{lr}
\hline \multicolumn{1}{c}{ Concepto } & Monto (pesos corrientes) \\
\hline Adecuación hospitalaria & 39.987 .308 \\
Ayuda humanitaria & 2.200 .000 .000 \\
Comunicación & 395.230 .641 \\
Dispositivos médicos & 6.981 .805 .365 \\
Logística & 1.000 .000 .000 \\
Otros & 1.644 .717 .078 \\
Pruebas COVID-19 & 1.051 .321 .882 \\
Publicidad & 627.000 .000 \\
Total general & $\mathbf{1 3 . 9 4 0 . 0 6 2 . 2 7 4}$ \\
\hline
\end{tabular}

\section{Discusión}

La intervención del Estado ante una emergencia del tamaño de COVID-19 es muy difícil de evaluar. En primer lugar, porque existen muchos aspectos derivados de esta pandemia que son inciertos, no solamente en la dimensión económica, sino también en el campo de la salud, la política, las instituciones, la educación, etc. En segundo lugar, porque no se cuenta con estrategias preestablecidas que deban seguir los países; algo que también se traslada a los gobiernos subnacionales.

Por lo anterior, los resultados alcanzados también se enfrentan a diferentes tipos de dictámenes, que además son relativos dependiendo de la perspectiva desde la cual se examine el fenómeno. En este punto, habría dos tipos de posiciones: por una parte, la postura gubernamental, que tiende a minimizar los efectos negativos de la pandemia, y por otra, la de la población o comunidad. Así entonces, las valoraciones, en los dos casos, tienden a ser divergentes.

En Colombia, el Gobierno Nacional ha venido defendiendo la idea de que la gestión de la pandemia ha sido correcta, no solamente en el campo de la salud, sino también en lo económico y social(23). No obstante, lo que se argumenta en este estudio es que la intervención fiscal ha sido insuficiente para las dimensiones que tiene la crisis. Incluso, en el contexto latinoamericano, Colombia no está entre los países 
que han destinado una mayor proporción del gasto público (\%PIB) a atender la pandemia(16).

Frente a la situación descrita, incluso las entidades multilaterales, como el Fondo Monetario Internacional (FMI), han insistido en la necesidad de que las medidas sean más agresivas, entendiendo la profundidad de la crisis tanto para los países desarrollados, como para las economías en desarrollo. Al respecto, la directora del FMI, Kristalina Georgieva, instó a los gobiernos y a los bancos centrales a que "...pongan en marcha todos los mecanismos posibles para contener los estragos económicos y humanos que la pandemia del coronavirus está causando. Más gasto en sanidad, más ayudas directas a las familias, más solidaridad con los países pobres. Más munición, por tierra mar y aire..." ${ }^{\prime 24) .}$

El comportamiento de los indicadores socioeconómicos refleja una situación muy difícil para Colombia, que puede agudizarse en la medida en que se amplíe la duración de la pandemia. Según el Departamento Administrativo Nacional de Estadística (DANE), durante el mes de abril de 2020, 5.4 millones de personas perdieron el empleo, y la tasa de desempleo llegó al 19,8\%(25).

Asimismo, frente a abril de 2019, "la producción real de la industria manufacturera presentó una variación de $-35,8 \%$, las ventas reales de $-35,9 \%$ y el personal ocupado de -7,8\%"(26). Los indicadores sociales también muestran una situación muy difícil, incluso antes de la crisis sanitaria. De acuerdo con las últimas estadísticas reportadas por el DANE para el año 2018(27), el departamento de Nariño registró un índice de pobreza multidimensional del 33,5\%, una incidencia de la pobreza monetaria del $41,4 \%$, y un nivel de pobreza monetaria extrema del $12,7 \%$. Para el total nacional, los porcentajes para los dos últimos indicadores fueron del $27 \%$ y el $7,2 \%$, respectivamente; reflejando así una brecha importante con relación al departamento de Nariño.

Bajo este contexto, esta investigación sugiere que se debe y se puede adoptar una posición mucho más agresiva por parte del Gobierno nacional y las entidades territoriales. Al respecto, hay que tener en cuenta que las medidas de confinamiento en una situación en la que las familias han perdido gran parte de sus ingresos resulta muy peligrosa. Por tal razón, se advierte que el Gobierno no está aplicando las medidas que se requieren para enfrentar un problema de este tamaño. Además, dichas intervenciones han llegado de manera tardía.

En el caso del departamento de Nariño, es importante recordar que, durante los años 2008 y 2009 -como resultado de la crisis que generaron las pirámides-, se perdieron alrededor de 50 mil empleos; que solamente se recuperaron en un periodo de aproximadamente cinco años(25). Probablemente, se puede pensar que la situación derivada de la pandemia de COVID-19 es diferente, porque en aquella ocasión se perdió una gran cantidad de capital. A pesar de las diferencias con relación a la crisis de 2008, en esta ocasión también se prevé que suceda algo similar. De acuerdo con las estadísticas del DANE, en la ciudad de Pasto, para el periodo comprendido entre diciembre de 2019 y febrero de 2020, de los 187000 puestos de trabajo, 105000 son informales; para una tasa del $56 \%$, que se ubica por encima del $48 \%$ reportada para el nivel nacional (23 ciudades)(28).

En el escenario de la emergencia sanitaria, en el que se han perdido muchos puestos de trabajo, dichas personas no pueden acceder a recursos de crédito, ni tampoco están incluidas en los programas sociales del Gobierno Nacional.

Ante esta compleja situación que plantea la emergencia sanitaria, es perentorio tomar medidas para reducir el impacto socioeconómico que se ha causado(15). En adelante, se sintetizan algunas propuestas, las cuales se agrupan, en aquellas dirigidas a las empresas, y las que se orientan a atender la situación de las familias. Posteriormente, aquellas que se requieren implementar de manera inmediata, y las que se pueden emprender en el mediano plazo.

Sobre el caso de las empresas, la idea fundamental es que las ayudas deben ser más ambiciosas y rápidas(29). Es importante recalcar que las empresas han sido bastante golpeadas durante la pandemia, especialmente por los desajustes de sus balances, puesto que tienen que asumir los gastos corrientes sin la posibilidad, en muchos casos, de recibir ingresos(15,29,30). 
En el caso de las disposiciones adoptadas en materia crediticia, estas solamente apuntan a resolver el problema de liquidez; que es un aspecto importante, pero no el único. Al final, las empresas contraerían deudas para poder asumir sus obligaciones corrientes, lo cual no constituye un incentivo por parte del Gobierno hacia las empresas, en su objetivo de conservar las tasas de empleo. Además, en un futuro cercano, los problemas de liquidez también se van a convertir en dificultades de solvencia, dado que muchos de sus activos, incluidas las cuentas por cobrar, no las podrán recuperar, precisamente por el impago de otros actores.

Otra cuestión que se debe mencionar es que el financiamiento al que accederían las empresas sería para apalancar los gastos administrativos, más no los de inversión. Esto implica que, necesariamente, se sacrifique la capacidad productiva en el mediano plazo(29). Por otra parte, el flujo de recursos desde los bancos hacia las empresas no es ágil, por lo cual, el riesgo de que las empresas no logren acceder efectivamente a las líneas de crédito es bastante elevado.

Un aspecto adicional es que, dentro de los beneficiarios de las líneas de crédito prometidas por el Gobierno, no se encuentran incluidos los independientes (trabajadores por cuenta propia o autónomos), ni tampoco aquellas personas $\mathrm{y}$ empresas que desarrollan actividades en el marco de la informalidad. Nuevamente, este tipo de decisiones no son eficaces y su cobertura es muy reducida. Al respecto, lo que ha mostrado la experiencia de otras crisis, es que los efectos negativos es más difícil resarcirlos con el paso del tiempo.

En el caso de las empresas de salud, el mensaje que envió el Gobierno Nacional durante el mes de marzo de 2020 es que iba a hacer un desembolso ágil de recursos económicos, entendiendo las dificultades estructurales del sector. En este punto, inicialmente, se contempló un monto de 5 billones de pesos ${ }^{(17) . S i n}$ embargo, existen dos situaciones que inciden en este proceso: la primera es que el flujo de recursos hacia los prestadores de servicios no está garantizado, puesto que son las Entidades Promotoras de Salud (EPS) quienes administran los recursos. En este contexto, solamente una parte de estos fondos ha llegado efectivamente a las IPS. La segunda, está asociada a que no se trata de recursos nuevos, si no que el gobierno está pagando muchas de las deudas que se habían contraído anteriormente(31).

Por los motivos señalados, no se puede asumir que los recursos anunciados van a servir para mejorar las condiciones de prestación de servicios de salud. Así las cosas, lo único que se resuelve en el corto plazo es un problema de liquidez. Ahora bien, dado que las IPS están dejando de facturar durante el periodo de la emergencia, más adelante van a tener dificultades para asumir sus obligaciones. Además, hay que tener en cuenta que estas instituciones están incurriendo en mayores gastos por el incremento de los precios de los insumos y dispositivos hospitalarios, medicamentos, material de bioseguridad, además de las nuevas inversiones requeridas para atender la emergencia sanitaria. De esta manera, los prestadores de servicios de salud, fundamentalmente los públicos, en lugar de fortalecerse, están asumiendo un riesgo bastante elevado(31).

Desde la perspectiva abordada en este estudio, se evidencia la necesidad de fortalecer el sistema de salud, y particularmente a los prestadores públicos(14), dado que son actores fundamentales para la atención de pacientes afectados por el coronavirus. Además, durante el periodo de la pandemia, se ha reducido notablemente la prestación de servicios de salud, ante lo cual, las EPS argumentan que los mismos se difieren. Sin embargo, muchos de los servicios definitivamente no se prestan a los usuarios. Además, el solo hecho de diferir la prestación de los servicios, hace que haya una reducción de los costos para las EPS(31).

Asimismo, en el marco de la pandemia COVID-19, se hace necesario fortalecer la educación, entendiéndose como un servicio público fundamental. Al respecto, hay unanimidad en el sentido de que solamente la inversión en ciencia y tecnología, permite a los países afrontar de mejor manera este tipo de circunstancias(32). En esta pandemia, dicha situación ha quedado bastante clara.

Por otra parte, se requiere un mayor apoyo para el sector agropecuario y la industria manufacturera. En el primer caso, porque es fundamental garantizar la seguridad alimentaria(33). Y en el segundo, porque se ha demostrado en esta pandemia, que la distribución de insumos médicos que hace el mercado no es suficiente ni eficiente. La dependencia de las importaciones realizadas desde ciertos países, como 
Estados Unidos, Alemania, China y Corea del Sur, deja ver que la provisión de suministros y dispositivos médicos está sujeta a una serie inconvenientes que dificultan la gestión de la emergencia sanitaria(5).

Otro aspecto a examinar, es la necesidad de que el Gobierno Nacional gestione nuevas fuentes de financiamiento para asumir los gastos derivados de la pandemia. En este sentido, no debe existir temor a utilizar cierto tipo de instrumentos, llamados no convencionales, cuando muchos países desarrollados así como varios países en desarrollo ya lo están haciendo(34). En el caso de Colombia, esto podría suceder con el uso de las reservas internacionales y el financiamiento del Gobierno Central con recursos del Banco de la República.

La política monetaria debería ser más agresiva(12,29,34), de manera que el Banco de la República no continúe actuando sacrificando la economía por alcanzar el objetivo inflacionario(9). Es importante considerar que así como en determinados meses ha incrementado el precio de los bienes básicos, el resto de componentes de la canasta de bienes y servicios está mostrando el comportamiento contrario, con el agravante de que existe la posibilidad que se presente una deflación en los precios de los bienes y de los activos.

Tampoco debe haber temor por el incremento del déficit fiscal y el saldo de la deuda pública. Dada la magnitud de la pandemia, dicho fenómeno necesariamente se va a dar. El tema más importante es que se pueda atender con suficiencia las necesidades derivadas de la crisis. La experiencia en el último episodio de este tipo para Colombia, que se dio en 1999, es muy aleccionadora en este sentido. En aquel momento, el Gobierno Nacional decidió aplicar unas medidas muy restrictivas en materia fiscal, que al final redundaron en un crecimiento económico muy bajo entre los años 2000 y 2003(9).

En el caso del departamento de Nariño, los datos presentados hasta el momento evidencian que tanto los municipios como la Gobernación han venido realizando algunas inversiones para atender los gastos derivados de la pandemia de COVID 19. Sin embargo, se colige que dichas medidas son insuficientes, considerando las dificultades por las que atraviesan las familias y empresas en esta zona del país. Desde luego, el argumento en este punto va a ser que el espacio fiscal para los entes territoriales es muy estrecho. Al respecto, lo que se ha advertido es que, efectivamente, es el GNC el que tiene mayores competencias en materia tributaria(7). De hecho, las entidades territoriales no pueden crear impuestos adicionales, teniendo la posibilidad solamente de administrar los que ya existen; convirtiéndose en un aspecto crítico en la gestión de la pandemia(7). No obstante, teniendo en cuenta la amplia disponibilidad de recursos de regalías, resulta bastante paradójico que esta fuente de financiamiento no se haya utilizado masivamente para la atención de la emergencia sanitaria.

Más allá de las peculiaridades que está evidenciando esta crisis, un elemento a tener en cuenta es que los efectos sociales y económicos de la pandemia van a ser mucho más drásticos para aquellos países, y personas, que muestran un alto grado de vulnerabilidad, entre los que se incluye a América Latina en general, y a Colombia en particular $(4,9)$. Además, se resalta el hecho de que este no es un aspecto nuevo. La razón básica es que estos países cuentan con muchas restricciones históricas para hacer frente a las exigencias en el frente económico, social, institucional, de salud, educación, etc.; especialmente cuando se presentan choques externos negativos(35).

Nuevamente, los mayores efectos se concentran en los países y personas más desprotegidas. Dado que en el mundo no hay la suficiente coordinación entre países, uno de los temores tiene que ver con que los esfuerzos no se distribuyan. Al respecto, se ha insistido en que debe haber coordinación y solidaridad entre países y regiones, no solamente porque sea correcto, como lo afirma Bill Gates, sino también porque es una decisión inteligente $(36,37)$.

Siguiendo la lógica evolucionista, infortunadamente, en esta carrera a la que se han sumado los diferentes países termina ganando el más fuerte. El problema es ver cómo surge la solidaridad en medio de la voraz competencia entre países (37). Si no se garantiza un mínimo de solidaridad supranacional, el contagio puede continuar. Ahora bien, una de las lecciones que se ha derivado de la pandemia de COVID-19 es que no solamente aquellos países que tengan las capacidades económicas puedan salir rápidamente de esta situación; el resto, necesariamente deberán hacerlo.

A pesar de todas las críticas hacia la acción de los gobiernos en las últimas décadas, en medio de esta 
emergencia sanitaria, se ha resaltado la importancia del Estado y el papel que este puede jugar como articulador de la sociedad. Después de muchos años de haber desmantelado la intervención estatal, de haberle quitado varias de sus herramientas de actuación, particularmente durante los procesos de liberalización comercial y financiera que se dieron en América Latina a finales de las décadas de los ochenta e inicios de los noventa, nuevamente se ha remarcado su importancia en todos los ámbitos(32).

Nuevamente se destaca la importancia del papel que juega la ciencia y la tecnología, así como de la asignación de recursos para su desarrollo; por cuanto permite a los gobiernos y a la sociedad en general afrontar efectivamente este tipo de fenómenos, como se observa en muchos países donde las decisiones para enfrentar la pandemia de COVID-19 estuvieron guiadas por la opinión de los expertos y no la de los políticos, hecho que sin lugar a dudas, es un paso importante.

\section{Conclusiones}

El objetivo de esta investigación fue evaluar las medidas adoptadas por el gobierno nacional colombiano, por las entidades territoriales del departamento de Nariño ante los desafíos sociales y económicos asociados a COVID-19, tomando como referencia el periodo marzo-mayo de 2020. Desde la perspectiva metodológica, es claro que este propósito es muy complejo debido a que el fenómeno estudiado está en curso, y no hay certeza del momento en que va a finalizar. Por tanto, resulta muy difícil hacer una valoración de este tipo. Además, los efectos de muchas de las medidas de política pública solamente se podrán dimensionar en su totalidad hacia el futuro.

Los hallazgos aportados dentro de esta investigación muestran que el impacto socioeconómico generado por COVID-19 es muy significativo para Colombia, y particularmente para un departamento altamente vulnerable como lo es Nariño; situación que se refleja en los indicadores de pobreza, desempleo, informalidad, desigualdad, etc. En términos económicos, se prevé una caída importante de la producción, que es un factor que terminará agudizando los problemas estructurales de esta zona del país. Además, se espera que los efectos ocasionados en el tejido productivo persistan por un periodo bastante prolongado.
Con relación a la intervención del gobierno nacional colombiano $\mathrm{y}$ las entidades territoriales del departamento de Nariño para atender la emergencia sanitaria, las medidas adoptadas dejan ver que la respuesta se ha dado. Sin embargo, se observa un deterioro bastante significativo y acelerado en la mayoría de indicadores socioeconómicos. Por tal razón, se colige que la intervención ha sido insuficiente y tardía, debido a las limitaciones fiscales y las precauciones y temores de los gobiernos para adoptar medidas más amplias en materia de política macroeconómica.

Es importante recalcar que el tiempo que los gobernantes tarden en tomar decisiones, influirá en el curso de la crisis y en la salida de la misma.

\section{Conflicto de intereses}

El autor declara que no existen conflictos de interés.

\section{Referencias}

1. Foladori G, Delgado Wise R. Para comprender el impacto disruptivo de la COVID-19, un análisis desde la crítica de la economía política. Migr Desarro. 2020;18(34):161-78.

2. Comisión Económica para América Latina y el Caribe. Salud y economía: una convergencia necesaria para enfrentar el COVID-19 y retomar la senda hacia el desarrollo sostenible en América Latina y el Caribe. 2020 [citado 16 de septiembre de 2020]; Disponible en: https://repositorio.cepal.org//handle/11362/45840

3. Trincado Aznar E, Trincado Aznar E. COVID-19: Historia y pensamiento económico para un enemigo invisible [Internet]. Pozuelo de Alarcón: Instituto Complutense de Estudios Internacionales (ICEI); 2020 [citado 16 de septiembre de 2020]. Disponible en: https://www.ucm.es/icei/icei-papers-covid19

4. El País. Carmen Reinhart: "Para la recuperación plena del PIB 'per cápita' faltan al menos cinco años" [Internet]. EL PAÍS. 2020 [citado 17 de septiembre de 2020]. Disponible en: https://elpais.com/economia/2020-09-17/carmenreinhart-para-la-recuperacion-plena-del-pib-per-capitafaltan-al-menos-cinco-anos.html

5. Comisión Económica para América Latina y el Caribe. Informe sobre el impacto económico en América Latina y el Caribe de la enfermedad por coronavirus (COVID-19) [Internet]. CEPAL; 2020 [citado 15 de junio de 2020]. Disponible en: https://www.cepal.org/es/publicaciones/45602-informeimpacto-economico-america-latina-caribe-la-enfermedadcoronavirus-covid

6. Krugman P. La recesión de la Covid-19 ya está aquí [Internet]. EL PAÍS. 2020 [citado 15 de junio de 2020]. Disponible en: https://elpais.com/economia/negocio/202004-03/la-recesion-de-la-covid-19-ya-esta-aqui.html

7. Portillo-Riascos L. Balance preliminar de la aplicación de la ley 1176 de 2007. Tendencias. 2011;12(1):7-43.

8. Bonet-Morón J, Ricciulli-Marín D, Pérez-Valbuena GJ, GalvisAponte LA, Haddad EA, Araújo IF, et al. Impacto económico 
regional del Covid-19 en Colombia: un análisis insumoproducto [Internet]. Banco de la República de Colombia; 2020 [citado 16 de septiembre de 2020]. Disponible en: https://repositorio.banrep.gov.co/bitstream/handle/20.50 0.12134/9843/DTSERU_288.pdf

9. Portillo-Riascos L. Vulnerabilidad e inestabilidad macroeconómica de Colombia en el período 1990-2015 [Internet] [info:eu-repo/semantics/doctoralThesis]. [Madrid]: Universidad Complutense de Madrid; 2019 [citado 15 de junio de 2020]. Disponible en: https://eprints.ucm.es/56826/

10. Portillo-Riascos LH. The concept of macroeconomic vulnerability. A review of the literature. Soc Econ. 2020;(40):99-118.

11. SECOP [Internet]. Bogotá (Col): sistema Electrónico para la Contratación Pública [citado 2020 junio]. Disponible en: https://www.contratos.gov.co/consultas/inicioConsulta.do

12. Comisión Económica para América Latina y el Caribe. Enfrentar los efectos cada vez mayores del COVID-19 para una reactivación con igualdad: nuevas proyecciones [Internet]. 2020 [citado 16 de septiembre de 2020]. Disponible

en: https://www.cepal.org/sites/default/files/presentation/fil es/final_200714_version_revisada_abppt_informe_covid_5_15_julio.pdf

13. World Bank. Global Economic Prospects, June 2020 [Internet]. Washington, DC: World Bank Group; 2020 [citado 10 de junio de 2020]. Disponible en: https://openknowledge.worldbank.org/bitstream/handle/ 10986/33748/9781464815539.pdf

14. Comisión Económica para América Latina y el Caribe. América Latina y el Caribe ante la pandemia del COVID-19: efectos económicos y sociales | CEPAL |. 2020 [citado 16 de septiembre de 2020]; Disponible en: https://www.cepal.org/es/publicaciones/45337-americalatina-caribe-la-pandemia-covid-19-efectos-economicossociales

15. Comisión Económica para América Latina y el Caribe. El desafío social en tiempos del COVID-19 [Internet]. CEPAL; 2020 [citado 15 de junio de 2020]. Disponible en: https://www.cepal.org/es/publicaciones/45527-desafiosocial-tiempos-covid-19

16. Ávila R. Covid-19: ¿Qué tanta plata destina Colombia respecto a sus vecinos? [Internet]. El Tiempo. 2020 [citado 9 de junio de 2020]. Disponible en: https://www.eltiempo.com/economia/sectores/coronaviru s-analisis-de-recursos-invertidos-a-mitigar-la-pandemiaen-colombia-y-la-region-488654

17. El Tiempo. Las cuentas de la cuarentena que llevó Minhacienda al Congreso [Internet]. El Tiempo. 2020 [citado 16 de septiembre de 2020]. Disponible en: https://www.eltiempo.com/economia/sectores/coronaviru s-en-colombia-cuentas-de-la-cuarentena-que-llevominhacienda-al-congreso-488040

18. El Tiempo. Gobierno espera contracción económica del $5,5 \%$ este año [Internet]. Portafolio.co. 2020 [citado 17 de septiembre de 2020]. Disponible en: https://www.portafolio.co/economia/gobierno-esperacontraccion-economica-del-5-5-este-ano-por-la-covid542144

19. El Tiempo. Corte Constitucional tumba el impuesto solidario [Internet]. El Tiempo. 2020 [citado 20 de septiembre de 2020].

Disponible

en: https://www.eltiempo.com/justicia/cortes/corteconstitucional-tumba-el-impuesto-solidario-526256

20. El Espectador. Se cayó el decreto que cambió las reglas pensionales en la pandemia [Internet]. ELESPECTADOR.COM. 2020 [citado 20 de septiembre de 2020]. Disponible

en: https://www.elespectador.com/noticias/judicial/se-cayoel-decreto-que-cambio-las-reglas-pensionales-en-lapandemia/

21. Departamento Administrativo de la Presidencia de la República. Decreto 513 del 2 de abril de 2020.pdf [Internet]. 2020 [citado 20 de septiembre de 2020]. Disponible en: https://dapre.presidencia.gov.co/normativa/normativa/DE CRETO\%20513\%20DEL\%202\%20DE\%20ABRIL\%20DE\%2 02020.pdf

22. Departamento Nacional de Planeación. Estado Presupuestal SGR [Internet]. 2020 [citado 12 de junio de 2020]. Disponible en: https://sicodis.dnp.gov.co/Reportes/EstadoPres upuestalSGR_v6.aspx

23. El País. Duque defendió su gestión de la pandemia y anunció más ayudas para afectados [Internet]. 2020 [citado 17 de septiembre de 2020]. Disponible en: https://www.elpais.com.co/colombia/duque-defendio-sugestion-de-la-pandemia-y-anuncio-mas-ayudas-paraafectados.html

24. El País. La directora del FMI, a Gobiernos y bancos centrales: "Gasten cuanto puedan, pero guarden los recibos"| Economía | EL PAÍS [Internet]. [citado 10 de junio de 2020]. Disponible en: https://elpais.com/economia/2020-04-15/georgievafmi-a-gobiernos-y-bancos-centrales-gasten-cuanto-puedanpero-guarden-los-recibos.html

25. Departamento Administrativo Nacional de Estadística (DANE). Empleo y desempleo [Internet]. [citado 8 de junio de 2020]. Disponible https://www.dane.gov.co/index.php/estadisticas-portema/mercado-laboral/empleo-y-desempleo

26. Departamento Administrativo Nacional de Estadística (DANE). Encuesta mensual manufacturera con enfoque territorial (EMMET) [Internet]. [citado 17 de junio de 2020]. Disponible en: https://www.dane.gov.co/index.php/estadisticas-portema/industria/encuesta-mensual-manufacturera-conenfoque-territorial-emmet

27. Departamento Administrativo Nacional de Estadística (DANE). Pobreza Monetaria y Multidimensional en Colombia 2018 [Internet]. [citado 17 de junio de 2020]. Disponible en: https://www.dane.gov.co/index.php/estadisticas-portema/pobreza-y-condiciones-de-vida/pobreza-ydesigualdad/pobreza-monetaria-y-multidimensional-encolombia-2018\#pobreza-por-departamentos-2018

28. Departamento Administrativo Nacional de Estadística (DANE). Empleo y desempleo [Internet]. [citado 17 de junio de 2020]. Disponible en: https://www.dane.gov.co/index.php/estadisticas-portema/mercado-laboral/empleo-y-desempleo

29. Comisión Económica para América Latina y el Caribe. Sectores y empresas frente al COVID-19: emergencia y reactivación [Internet]. CEPAL; 2020 [citado 16 de septiembre de 2020]. Disponible en: https://www.cepal.org/es/publicaciones/45734-sectoresempresas-frente-al-covid-19-emergencia-reactivacion 
30. Confecámaras (Confederación Colombiana de Cámaras de Comercio). Encuesta Cámaras de Comercio consolidada.pdf [Internet]. 2020 [citado 16 de septiembre de 2020]. Disponible en: http://www.confecamaras.org.co/phocadownload/2020/E ncuesta\%20C\%C3\%A1maras\%20de\%20Comercio\%20cons olidada.pdf

31. Semana. ¿Por qué se están quebrando los hospitales? [Internet]. Semana.com Últimas Noticias de Colombia y el Mundo. 2020 [citado 16 de septiembre de 2020]. Disponible en:

https://www.semana.com/nacion/articulo/coronavirusen-colombia-clinicas-y-hospitales-enfrentan-problemasfinancieros/666028/

32. González A. El Estado ha llegado a la economía para quedarse | Negocios | EL PAÍS [Internet]. [citado 16 de septiembre de 2020]. Disponible en: https://elpais.com/economia/202004-25/el-estado-ha-llegado-para-quedarse-en-laeconomia.html

33. Comisión Económica para América Latina y el Caribe. Cómo evitar que la crisis del COVID-19 se transforme en una crisis alimentaria: acciones urgentes contra el hambre en América Latina y el Caribe [Internet]. CEPAL; 2020 [citado 17 de septiembre de 2020]. Disponible en: https://www.cepal.org/es/publicaciones/45702-como- evitar-que-la-crisis-covid-19-se-transforme-crisisalimentaria-acciones

34. López 0 . ¿Hay espacio para medidas de política monetaria no convencionales en América Latina? | CAF [Internet]. 2020 [citado 17 de septiembre de 2020]. Disponible en: https://www.caf.com/es/conocimiento/visiones/2020/05/ hay-espacio-medidas-de-politica-monetaria-noconvencionales-en-america-latina/

35. Portillo-Riascos L. Los orígenes de la vulnerabilidad macroeconómica y sus principales efectos: propuesta de un marco teórico. [Internet]. Iberoamerican Journal of Development Studies; 2020. Disponible en: http://ried.unizar.es/public/abstracts/losorigenesdelavuln erabilidad.pdf

36. Gates B. Tribuna | Una estrategia mundial contra la Covid-19. El País [Internet]. 12 de abril de 2020 [citado 18 de junio de 2020]; Disponible https://elpais.com/elpais/2020/04/11/opinion/15866007 30_628755.html

37. El País. Edgar Morin: "Vivimos en un mercado planetario que no ha sabido suscitar fraternidad entre los pueblos" [Internet]. EL PAÍS. 2020 [citado 15 de junio de 2020]. Disponible en: https://elpais.com/cultura/2020-0411/edgar-morin-vivimos-en-un-mercado-planetario-queno-ha-sabido-suscitar-fraternidad-entre-los-pueblos.html 